\title{
Group IV Platforms for the Mid-Infrared
}

\author{
G. Z. Mashanovich ${ }^{1}$, M. Nedeljkovic ${ }^{1}$, X. Chen $^{1}$, T. M. Ben Masaud ${ }^{2}$, M. Muneeb ${ }^{3}$, M. Strain ${ }^{4}$, \\ M. Sorel ${ }^{4}$, T. F. Krauss ${ }^{5}$, G. C. Roelkens ${ }^{3}$, A. C. Peacock ${ }^{1}$, H. M. H. Chong ${ }^{2}$, G. T. Reed ${ }^{1}$ \\ ${ }^{1}$ Optoelectronics Research Centre, University of Southampton, Southampton, SO17 1BJ, UK \\ ${ }^{2}$ Electronics and Computer Science, University of Southampton, Southampton, SO17 1BJ, UK \\ ${ }^{3}$ Photonics Research Group - NB-Photonics, Ghent University-IMEC, B-9000 Ghent, Belgium \\ ${ }^{4}$ School of Engineering, University of Glasgow, Glasgow G12 8LT, UK \\ ${ }^{5}$ Department of Physics, University of York, Heslington, York, YO10 5DD, UK
}

\begin{abstract}
We have investigated several material platforms for the mid-infrared including silicon on insulator (SOI), polycrystalline silicon, and suspended silicon structures. We review photonic devices based on these platforms including splitters, ring/racetrack resonators, Mach-Zehnder interferometers, and spectrometers.
\end{abstract}

Keywords: Mid-infrared, silicon, silicon-on-insulator, Mach-Zehnder interferometer, multimode interference, ring resonator, polycrystalline silicon.

\section{INTRODUCTION}

Recently, the mid-infrared (MIR) group IV photonics has attracted interest from several research groups due to a host of important application areas, such as sensing, medical diagnostics, industrial control, communications, defence and security $[1,2]$. Particularly important is the so-called 'fingerprint' region where high selectivity sensing can be achieved. Integrated, compact, low cost and low energy solutions in group IV materials will have significant advantages over expensive and bulky spectrometers currently used. Such devices could be used as breath analysers in disease diagnostics, for environmental monitoring, or for toxic and explosive sensing. To address the "explosion" of internet traffic, low loss optical fibres in the $2-3 \mu \mathrm{m}$ are being actively researched to increase the current capacity [3]. Low cost and low energy group IV integrated circuits that would comprise both active and passive photonic components may find application in nodes of such networks.

In the last 3-4 years, non-linear effects in the short-wave range have been reported [e.g. 4-7], as well as passive devices in SOI [8-13], Si on porous Si (SiPSi) [4], SOS [14-17], and Ge-on-Si [18]. Moreover, active devices are being integrated on SOI platform for 2-2.5 $\mathrm{m}$ [19-22]. In this paper we review our recent work on different group IV materials: SOI, suspended $\mathrm{Si}$, and polycrystalline $\mathrm{Si}$. We also review demonstrated passive components, including multimode interference (MMI) splitters, ring resonators, Mach-Zehnder interferometers (MZIs), and spectrometers.

\section{WAVEGUIDES}

\subsection{SOI waveguides}

As $\mathrm{SiO}_{2}$ has high material loss at 2.6-2.9 $\mu \mathrm{m}$ and beyond 3.6 $\mu \mathrm{m}$ [23] we have investigated SOI viability in the MIR, particularly in the $3-4 \mu \mathrm{m}$ range. We first reported propagation loss measurements of SOI rib waveguides at $\lambda=3.39 \mu \mathrm{m}$, with the following dimensions: width $2 \mu \mathrm{m}$, height $2 \mu \mathrm{m}$, etch depth $1.2 \mu \mathrm{m}$, BOX thickness $2 \mu \mathrm{m}$ [8]. The propagation loss of the waveguides was $0.6 \pm 0.2 \mathrm{~dB} / \mathrm{cm}$. We have characterised the same waveguides at the wavelengths of $3.73 \mu \mathrm{m}$ and $3.80 \mu \mathrm{m}$, for which the measured losses were $1.5 \pm 0.2 \mathrm{~dB} / \mathrm{cm}$ and $1.8 \pm 0.2 \mathrm{~dB} / \mathrm{cm}$, respectively [17]. It can be seen that the propagation loss increases quite significantly over this wavelength range. Part of this increase can be accounted for by the increased optical mode interaction with the sidewalls, though the remainder reflects the increased absorption in $\mathrm{SiO}_{2}$ for $\lambda>3.6 \mu \mathrm{m}$. 
Strip waveguides with smaller dimensions can have low loss for much smaller bend radii than larger rib waveguides, hence tighter integration of components on chip for a smaller device footprint is possible. However, because of the greater interaction of the optical mode with both the sidewalls, and the BOX layer, there is larger propagation loss. We have fabricated and characterised SOI strip waveguides with $\mathrm{H}=500 \mathrm{~nm}$, a $3 \mu \mathrm{m}$ BOX layer thickness, and width variations of $1.0 \mu \mathrm{m}, 1.2 \mu \mathrm{m}$ and $1.4 \mu \mathrm{m}$, for which we have reported the results in [9]. The minimum propagation loss was $\sim 4.6 \mathrm{~dB} / \mathrm{cm}$. In our recent work we have improved the loss to $\sim 3 \mathrm{~dB} / \mathrm{cm}$ at $3.74 \mu \mathrm{m}, \sim 3.5 \mathrm{~dB} / \mathrm{cm}$ at $3.8 \mu \mathrm{m}$, and $<4 \mathrm{~dB} / \mathrm{cm}$ at $3.84 \mu \mathrm{m}$ for $400 \mathrm{~nm}$ high SOI waveguides fabricated in a CMOS pilot line at IMEC [24]. As a $2 \mu \mathrm{m}$ BOX was used, the substrate leakage contributed by $0.6-0.8 \mathrm{~dB} / \mathrm{cm}$, hence we expect the loss can be reduced further if a $3 \mu \mathrm{m}$ thick BOX is used in the SOI.

\subsection{PhC waveguides}

By using a $500 \mathrm{~nm}$ SOI with $3 \mu \mathrm{m}$ BOX we have recently demonstrated photonic crystal (PhC) waveguides in the 2.9 to $3.9 \mu \mathrm{m}$ wavelength range [10]. We have chosen the well-known W1 design, which consists of a two-dimensional hexagonal $\mathrm{PhC}$ lattice of air holes in a silicon slab with one row of holes removed. A number of different W1 PhC waveguides with varying lattice periods $\mathrm{a}=1040-1120 \mathrm{~nm}$ and hole radii $\mathrm{r} / \mathrm{a}=0.26-0.28$ were fabricated, targeting guided modes between $2.9 \mu \mathrm{m}$ and $3.9 \mu \mathrm{m}$. The waveguides were fabricated using e-beam lithography, RIE etching and HF removal of buried oxide.

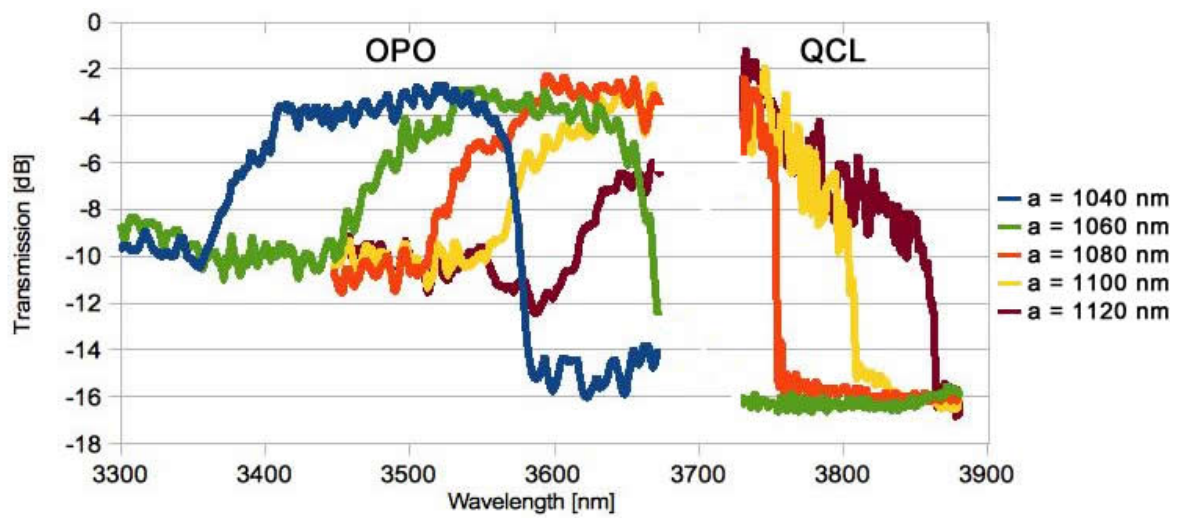

Figure 1. Transmission through photonic crystal waveguides [10]

The waveguides were characterised using an OPO and a QCL thus covering a large wavelength range. The combined measurements, normalised to access waveguides, are shown in Figure 1. Clear short and long wavelength cut-offs that correspond to the position of the light line and the $\mathrm{PhC}$ bandgap, respectively, are apparent in Figure 1.

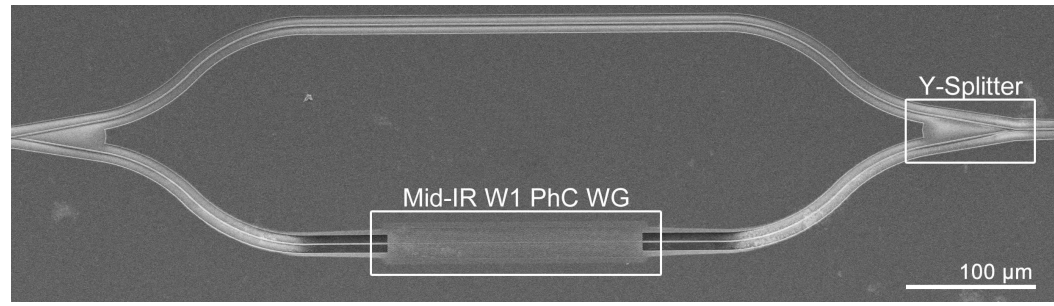

Figure 2. SEM images of an integrated unbalanced MIR MZI used to measure the group index of the PhC waveguides [10]

To measure the group index, we lithographically integrated an unbalanced Mach-Zehnder interferometer (MZI) onto the chip (Figure 2) and scanned the wavelength in order to measure the interference pattern without the need for an OSA or an external interferometer. The MZI is formed by utilizing two single mode 50:50 y-splitters and placing a PhC waveguide in one arm of the MZI (Figure 2). The measurement was limited to a group index value of $n_{\mathrm{g}} \approx 12$ by the relatively large effective linewidth of the OPO. This limitation can be addressed by using dispersion engineered slow light waveguides, which will allow access to much higher group index values and correspondingly will allow the 
observation of nonlinear effects. We have also measured the propagation loss by using the cut back method. A value of $20 \mathrm{~dB} / \mathrm{cm}$ was obtained. We believe that this figure can be significantly reduced by tuning the etching process and reducing the sidewall roughness.

\subsection{Suspended waveguides}

To extend the transmission of the SOI platform, suspended waveguides can be used. Cheng at al. designed and characterised 340nm high Si rib waveguides with an undercut BOX [11,12]. Deep periodical holes were etched close to the rib waveguides, enabling removal of the BOX in HF solution. The holes were located far enough from the rib to have negligible interaction with the optical mode. The propagation loss of $3 \mathrm{~dB} / \mathrm{cm}$ was measured at $2.75 \mu \mathrm{m}$.
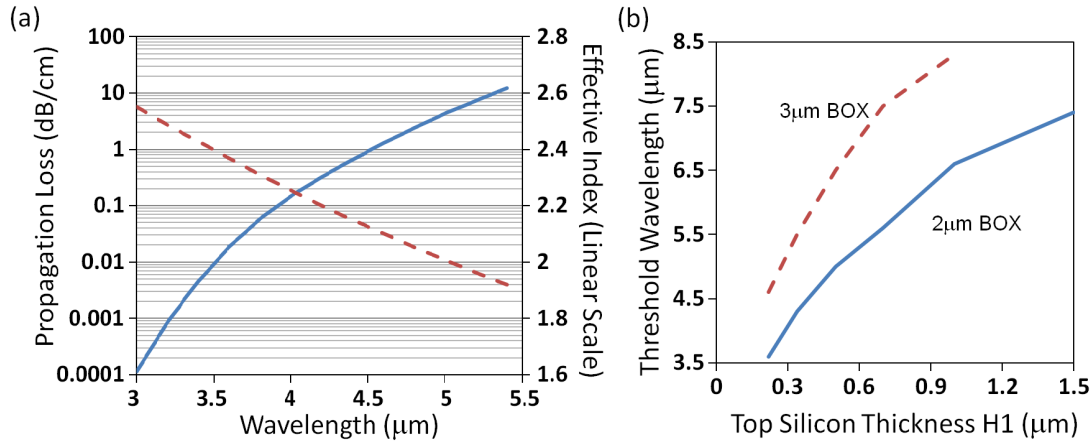

Figure 3. (a) Propagation loss and effective index of a suspended waveguide on SOI wafer with 340nm top silicon and $2 \mu \mathrm{m}$ BOX; (b) cut-off wavelength for suspended waveguides on SOI vs top silicon for $2 \mu \mathrm{m}$ and $3 \mu \mathrm{m}$ BOX thicknesses [25]

We have theoretically investigated the transmission range of such waveguides [25]. Figure 3a shows the propagation loss and effective index of the waveguides with $340 \mathrm{~nm}$ top silicon and $2 \mu \mathrm{m}$ BOX thickness. Such waveguides can support wavelengths up to $4.3 \mu \mathrm{m}$. We then plotted the maximum transmission wavelengths for different top silicon and BOX thicknesses in Figure 3b. For example, suspended waveguides on 500nm SOI with $3 \mu \mathrm{m}$ BOX can support wavelengths up to $6.5 \mu \mathrm{m}$.

\subsection{Poly Si-on-SOI waveguides}

Very recently, we have investigated poly Si on SOI waveguides (Figure 4). The waveguides were fabricated using the imecAP process: $5 \mathrm{~nm}$ of thermal $\mathrm{SiO}_{2}$ was grown on $220 \mathrm{~nm}$ SOI with $2 \mu \mathrm{m}$ buried oxide. Then $160 \mathrm{~nm}$ of amorphous $\mathrm{Si}$ (a-Si) was deposited using LPCVD. On top of the a-Si, 10nm of $\mathrm{SiO}_{2}$ and 70nm of $\mathrm{SiN}$ were deposited using PECVD. The SiN layer served as a hard mask for the waveguide etching. The stack was subsequently annealed at $750^{\circ} \mathrm{C}$ for 30 minutes to convert the a-Si to poly $\mathrm{Si}$. This step was preformed to increase the temperature budget for eventual postprocessing on the silicon wafer. The waveguides were fabricated using 193nm DUV lithography and halogen based dry etching [24].

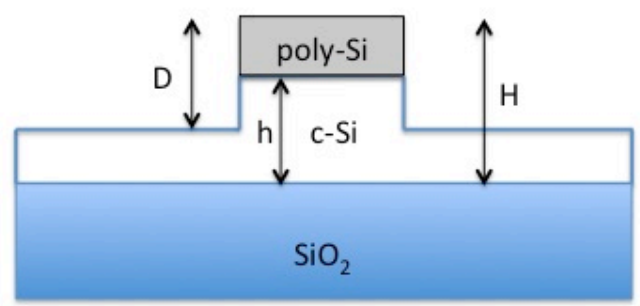

Si substrate

Figure 4. Schematic of a poly Si on SOI rib waveguide

Three different waveguide structures with $\mathrm{H}=380 \mathrm{~nm}$ were fabricated: 1) rib waveguide with $\mathrm{D}$ (etch depth) $=200 \mathrm{~nm}$ and $\mathrm{W}$ (wavegide width) $=1100 \mathrm{~nm}, 2)$ rib waveguide with $\mathrm{D}=160 \mathrm{~nm}$ and $\mathrm{W}=1350 \mathrm{~nm}$, and 3) rib waveguide with 2 aligned 
etch steps, $160 \mathrm{~nm}$ poly $\mathrm{Si}$ etch $\left(\mathrm{W}_{1}=1150 \mathrm{~nm}\right)$ and $220 \mathrm{~nm}$ crystalline $\mathrm{Si}$ etch $\left(\mathrm{W}_{2}=1450 \mathrm{~nm}\right)$. The cut back method was used to measure the propagation loss of these waveguides. At the wavelength of $3.76 \mu \mathrm{m}$ we have measured the following propagation losses for the three waveguides respectively: $7.6 \mathrm{~dB} / \mathrm{cm}, 5.3 \mathrm{~dB} / \mathrm{cm}$ and $5.8 \mathrm{~dB} / \mathrm{cm}$ [24]. We attribute these larger losses than those obtained for the crystalline Si waveguides discussed in section 2.1 to the scattering loss in the poly Si layer.

\section{SPLITTERS, INTERFEROMETERS AND SPECTROMETERS}

In this section passive devices based on submicron Si layer thickness are presented. We review devices based on 500nm $\mathrm{Si}, 400 \mathrm{~nm} \mathrm{Si}$, and $380 \mathrm{~nm}$ poly $\mathrm{Si} /$ crystalline $\mathrm{Si}$ waveguides.

\section{1. $1 \times 2$ Multimode interferometers}

Multimode interferometers are devices that work on the principal of self-imaging in a wide multimode waveguide, and are used as splitters or couplers for different numbers of input and output waveguides [26]. Figure 5 shows an SEM image of one of the fabricated MMIs, with the design variables marked on the image. Prior to the fabrication, detailed 3D simulations of the device were performed using the Photon Design Fimmprop software package.

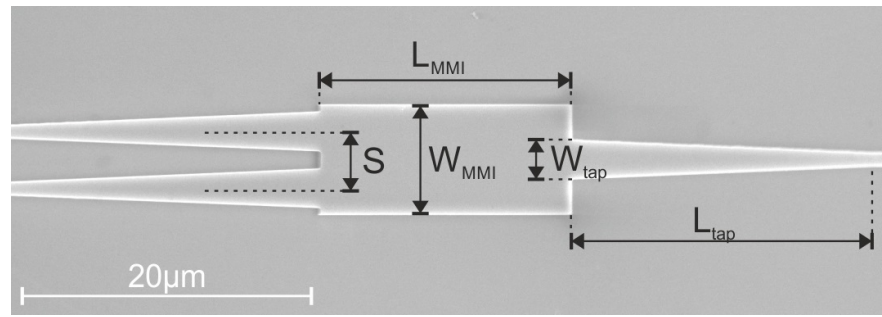

Figure 5. SEM image of fabricated $1 \times 2$ MMI with tapered input and output ports.

The fabricated devices were based on both $500 \mathrm{~nm}$ strip and $400 \mathrm{~nm}$ rib waveguide designs. For the device with $500 \mathrm{~nm} \mathrm{Si}$ layer thickness, the designed dimensions were $\mathrm{W}_{\mathrm{MMI}}=8 \mu \mathrm{m}, \mathrm{L}_{\mathrm{MMI}}=21.83 \mu \mathrm{m}, \mathrm{W}_{\text {tap }}=3.0 \mu \mathrm{m}, \mathrm{L}_{\text {tap }}=20 \mu \mathrm{m}$ and $\mathrm{S}=4.0 \mu \mathrm{m}$, with simulations predicting an insertion loss of $0.11 \mathrm{~dB}$ (surface scattering loss neglected). For the device with $400 \mathrm{~nm} \mathrm{Si}$ layer thickness, the designed dimensions were $\mathrm{W}_{\mathrm{MMI}}=8 \mu \mathrm{m}, \mathrm{L}_{\mathrm{MMI}}=21.0 \mu \mathrm{m}, \mathrm{W}_{\text {tap }}=2.6 \mu \mathrm{m}, \mathrm{L}_{\text {tap }}=20 \mu \mathrm{m}$ and $\mathrm{S}=4.18 \mu \mathrm{m}$, with simulations predicting an insertion loss of $0.08 \mathrm{~dB}$. The insertion loss of the fabricated devices was measured by chaining different number of pairs of MMIs and measuring the transmission through them. The measured loss for the $500 \mathrm{~nm}$ Si design was $0.29 \pm 0.17 \mathrm{~dB} / \mathrm{MMI}$, and for the $400 \mathrm{~nm}$ Si $0.25 \pm 0.02 \mathrm{~dB} / \mathrm{MMI}$. Figure 6 shows the transmission plotted against number of MMIs from which the loss figure was calculated [27]. The loss figures for both of these devices rival the best achieved loss figures for NIR silicon photonics [e.g. 28, 29].

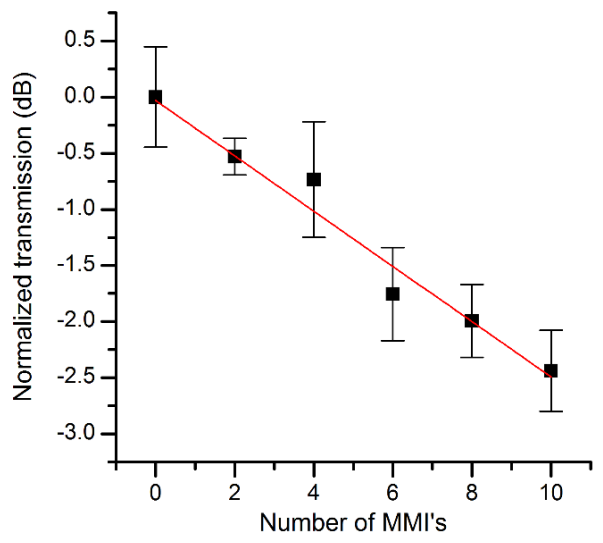

Figure 6. Normalized transmission vs number of MMIs for $\mathrm{H}=400 \mathrm{~nm}, \mathrm{D}=350 \mathrm{~nm}, \mathrm{~W}_{\mathrm{MMI}}=8 \mu \mathrm{m}, \mathrm{L}_{\mathrm{MMI}}=21.0 \mu \mathrm{m}$, $\mathrm{W}_{\text {tap }}=2.6 \mu \mathrm{m}, \mathrm{L}_{\text {tap }}=20 \mu \mathrm{m}$ and $\mathrm{S}=4.18 \mu \mathrm{m}$. The slope of the graph gives the insertion loss/MMI of $0.25 \pm 0.02 \mathrm{~dB} / \mathrm{MMI}$ [27]. 


\subsection{Racetrack and ring resonators}

Ring/racetrack resonators are used as wavelength filters and for converting a phase change into an amplitude change in either modulators or sensors. We have demonstrated racetrack resonators based on 500nm high strip waveguides [9]. The devices had a bending radius of $100 \mu \mathrm{m}$, a coupling region length of $50 \mu \mathrm{m}$, and an edge to edge spacing between the bus waveguide and ring waveguide of $1.2 \mu \mathrm{m}$. They were treated by thermal oxidation to reduce surface roughness. The transmission spectrum of the racetrack resonator was measured using the tunable QCL. The FSR of the device was measured to be $4.12 \mathrm{~nm}$ with an associated group index of 4.64, compared to theoretical values of FSR $=4.24 \mathrm{~nm}$, and $n_{g}$ $=4.53$. The experimentally measured resonance peaks exhibited an extinction ratio up to $10 \mathrm{~dB}$, and an average Q-factor of $8.2 \mathrm{k}$. From simulations the Q-factor was expected to be in the 30-40k range. This discrepancy may have arisen due to the QCL having mode-hopping behaviour, which could reduce the effective linewidth of the measurement, and be reflected in a lower Q-factor value.

\subsection{Mach-Zehnder interferometers}

Mach-Zehnder interferometers for the mid-IR have been reported in the literature [10] for use in a group index measurement of photonic crystals, and as a part of an optical modulator in silicon [21]. Neither paper investigated the characteristics of the MZI itself, so we have reported the extinction ratios and insertion losses of asymmetric MZIs based on both $400 \mathrm{~nm}$ and $500 \mathrm{~nm}$ high $\mathrm{Si}$ waveguides that have one input and one output, and that use the MMIs reported above as splitters and couplers [27].

Like ring resonators, Mach-Zehnder interferometers can be used as wavelength filters, or for converting a refractive index change into a change in optical intensity. The advantage of MZIs is that they are far less sensitive to thermal variations than rings, though they also typically have a much larger device footprint. The FSR of an asymmetric $1 \times 1$ MZI is given by:

$$
F S R=\frac{\lambda^{2}}{n_{g} \Delta L-\lambda}
$$

where $\Delta L$ is the arm length difference. Our MZIs with $400 \mathrm{~nm}$ thick Si waveguides were fabricated with $\Delta L=300 \mu \mathrm{m}$, and the MZIs with $500 \mathrm{~nm}$ thick waveguides had $\Delta L=350 \mu \mathrm{m}$. The transmission spectrum of the former is shown in Figure 7.

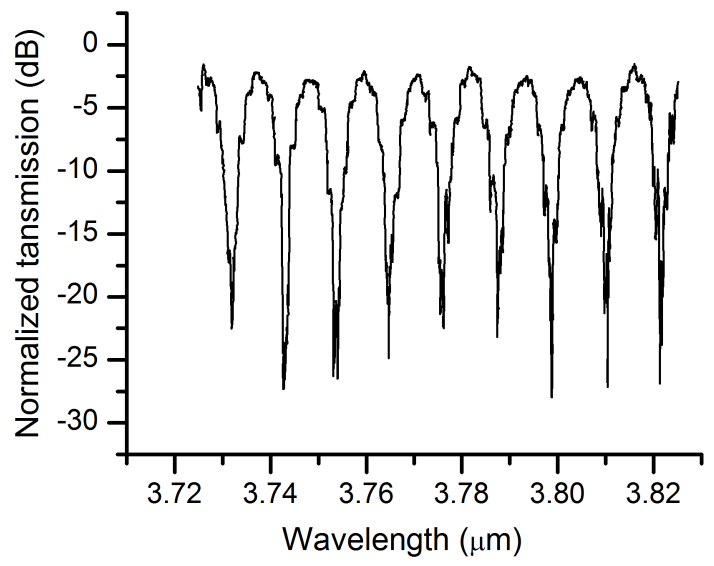

Figure 7. Normalized transmission spectrum of an MZI in SOI based on 400nm high rib waveguides with 350nm etch depth [27].

The MZIs exhibited extinction ratios up to $34 \mathrm{~dB}$ and insertion loss of $1.6-2.4 \mathrm{~dB}$.

\subsection{Spectrometers}

Recently, we have demonstrated arrayed waveguide gratings (AWGs) and echelle gratings based on 400nm crystalline $\mathrm{Si}$ and 380nm poly Si/crystalline Si waveguides [24]. Six channel AWGs (for the 3.7-3.8 $\mu \mathrm{m}$ range) were designed for TE polarised light with channel spacing of $10-12 \mathrm{~nm}$ and a total device size of $\sim 1 \times 0.7 \mathrm{~mm}^{2}$. The performance of the AWGs were close to the state-of-the-art SOI AWGs at telecommunications wavelengths (insertion loss of -1dB and cross talk $25 \mathrm{~dB}$ ). Eight channel echelle gratings had 57 grating facets, $10-12 \mathrm{~nm}$ channel spacing and an average size of 
$\sim 1.7 \times 1.4 \mathrm{~mm}^{2}$. To enhance the reflectivity of the grating facets, distributed Bragg reflectors (DBR) were implemented. The echelle gratings were also designed for TE polarisation. The insertion loss was again in the 1.5-2.5 $\mathrm{dB}$ range and crosstalk was less than $-15 \mathrm{~dB}$.

\section{SUMMARY}

We have investigated different group IV based material platforms for integrated photonics in the mid-infrared region by fabricating and characterising waveguides and other passive devices. Rib SOI waveguides had losses as low as 0.6$0.7 \mathrm{~dB} / \mathrm{cm}$ at $3.39 \mu \mathrm{m}$, that increased to $1.8 \mathrm{~dB} / \mathrm{cm}$ at $3.80 \mu \mathrm{m}$. Strip waveguides with $400 \mu \mathrm{m}$ height had propagation loss as low as $3 \mathrm{~dB} / \mathrm{cm}$ at $\lambda=3.8 \mu \mathrm{m}$. Poly $\mathrm{Si}$ on SOI waveguides showed $5-7 \mathrm{~dB} / \mathrm{cm}$ loss at $3.8 \mu \mathrm{m}$. We have also demonstrated low loss SOI MMIs, MZIs, AWG and echelle grating spectromenters in the MIR. These results show that SOI can be a viable material platform at wavelengths up to almost $4 \mu \mathrm{m}$. To enable propagation of MIR light in SOI at longer wavelengths, suspended structures can be used.

\section{ACKNOWLEDGEMENTS}

This work is supported by the Royal Society (Goran Mashanovich's URF “Mid-infrared Silicon Photonics") and by EPSRC through "UK Silicon Photonics" programme.

\section{REFERENCES}

[1] R. A. Soref, "Group IV photonics for the mid infrared," SPIE Photonics West 2013.

[2] R.A. Soref, "Mid-infrared photonics in silicon and germanium," Nature Photonics 4, 495-497 (2010).

[3] N. MacSuibhne, Z. Li, B. Baeuerle, J. Zhao, J. Wooler, S. Alam, F. Poletti, M. Petrovich, A. Heift, I. Giles, B. Palsdottir, L. Gruner-Nielsen, R. Phelan, J. O’Caroll, B. Kelly, D. Murphy, A. Ellis, D. Richardson, F. Garcia Gunning, "Wavelength division multiplexing at 2um," PDP Th3A3, ECOC 2012.

[4] S. Zlatanovic, J. S. Park, S. Moro, J. M. Chavez-Boggio, I. V. Divliansky, N. Alic, S. Mookherjea, and S. Radic, "Mid-infrared wavelength conversion in silicon waveguides using ultracompact telecom-band-derived pump source," Nature Photonics, vol. 4, pp. 561-564, 2010.

[5] X. Liu, R. M. Osgood, Y. A. Vlasov, and W. M. J. Green, "Mid-infrared optical parametric amplifier using silicon nanophotonic waveguides," Nature Photonics, vol. 4, pp. 557-560, 2010.

[6] X. Liu, B. Kuyken, G. Roelkens, R. Baets, R. M. Osgood Jr., W. M. J. Green, "Bridging the mid-infrared-totelecom gap with silicon nanophotonic spectral translation", Nature Photonics, vol. 6, pp. 667-671, 2012.

[7] B. Kuyken, X. Liu, R. M. Osgood Jr., R. Baets, G. Roelkens, W. M. J. Green, "A silicon-based widely tunable short-wave infrared optical parametric oscillator," Optics Express, vol. 21, pp. 5931-5940, 2013.

[8] G. Z. Mashanovich, M. M. Milosevic, M. Nedeljkovic, N. Owens, B. Xiong, E. J. Teo, and Y. Hu, "Low loss silicon waveguides for the mid-infrared," Opt. Express 19(8), $7112-7119$ (2011).

[9] M. M. Milosevic, M. Nedeljkovic, T.-B. Masaud, E. Jaberansary, H. M. H. Chong, N. G. Emerson, G. T. Reed, and G. Z. Mashanovich, "Silicon waveguides and devices for the mid-infrared," Appl. Phys. Lett. 101, 121105 (2012).

[10] C. Reimer, M. Nedeljkovic, D. J. M. Stothard, M. O. S. Esnault, C. Reardon, L. O'Faolain, M. Dunn, G. Z. Mashanovich, and T. F. Krauss, "Mid-infrared photonic crystal waveguides in silicon," Opt. Express 20, 2936129368 (2012).

[11] Z. Cheng, X. Chen, C. Y. Wong, K. Xu, and H. K. Tsang, "Mid-infrared suspended membrane waveguide and ring resonator on silicon-on-insulator," IEEE Phot. J. 4, 1510-1519 (2012).

[12] Z. Cheng, X. Chen, C. Y. Wong, K. Xu, K. Y. Fung, Y. M. Chen, H. K. Tsang, "Focusing subwavelength grating coupler for mid-infrared suspended membrane waveguide," Optics Letters, vol. 37, pp. 1217-1219, 2012.

[13] N. Hattasan, B. Kuyken, F. Leo, E.M.P. Ryckeboer, D. Vermeulen, G. Roelkens, "High-efficiency SOI fiber-tochip grating couplers and low-loss waveguides for the short-wave infrared," IEEE Photon. Technol. Lett., vol. 24, pp.1536-1538, 2012.

[14] R. Shankar, I. Bulu, and M. Lončar, "Integrated high-quality factor silicon-on-sapphire ring resonators for the midinfrared," Appl. Phys. Lett., vol. 102, 051108, 2013.

[15] T. Baehr-Jones, A. Spott, R. Ilic, B. Penkov, W. Ashder, and M. Hochberg, "Silicon-on-sapphire integrated waveguides for the mid-infrared," Opt. Express 18(12), 12127-12135 (2010). 
[16] F. Li, S. Jackson, C. Grillet, E. Magi, D. Hudson, S. J. Madden, Y. Moghe, C. O’Brien, A. Read, S. G. Duvall, P. Atanackovic, B. J. Eggleton and D. Moss, "Low propagation loss silicon-on-sapphire waveguides for the midinfrared," Opt. Express 19(16), 15212-15220 (2011).

[17] M. M. Milošević, D. J. Thomson, X. Chen, D. Cox, and G. Z. Mashanovich, "Silicon waveguides for the 3-4 $\mu \mathrm{m}$ wavelength range," 8th IEEE Group IV Photonics conference, London, UK, (2011).

[18] Y-C Chang, V. Paeder, L. Hvozdara, J-M Hartmann, and H.P. Herzig, "Low-loss germanium strip waveguides on silicon for the mid-infrared," Opt. Letters 37, 2883-2885 (2012).

[19] E. M. P. Ryckeboer, A. Gassenq, M. Muneeb, N. Hattasan, S. Pathak, L. Cerutti, J.-B. Rodriguez, E. Tournie, W. Bogaerts, R. Baets, G. Roelkens, Silicon-on-insulator spectrometers with integrated GaInAsSb photodiodes for wide-band spectroscopy from 1510 to $2300 \mathrm{~nm}$, Optics Express, vol. 21, pp. 6101-6108, 2013.

[20] A. Gassenq, N. Hattasan, E. M. P. Ryckeboer, J. B. Rodriguez, L. Cerutti, E. Tournie, G. Roelkens, "Study of evanescently-coupled and grating-assisted GaInAsSb photodiodes integrated on a silicon photonic chip," Optics Express, vol. 20, pp. 11665-11672, 2012.

[21] M. A. Van Camp, S. Assefa, D. M. Gill, T. Barwicz, S. M. Shank, P. M. Rice, T. Topuria, and W. J. Green, "Demonstration of electrooptic modulation at $2165 \mathrm{~nm}$ using a silicon Mach-Zehnder interferometer," Opt. Express 20, 28009-28016 (2012).

[22] N. Hattasan, A. Gassenq, L. Cerutti, J.B. Rodriguez, E. Tournie, G. Roelkens, "The study of integrated thin film GaSb-based Fabry Perot lasers: towards a fully integrated spectrometer on a Silicon-On-Insulator waveguide circuit," SPIE Photonics West 2013.

[23] E. D. Palik, Handbook of Optical Constants of Solids, Vol. 1, London: Academic, London, 1985.

[24] M. Muneeb, X. Chen, P. Verheyen, S. Pathak, A. Malik, M. Nedeljkovic, J. Van Campenhout, G. Z. Mashanovich, and G. Roelkens, "Demonstration of silicon-on-insulator mid-infrared spectrometers operating at 3.8 $\mathrm{mm}$," Optics Express 2013.

[25] X. Chen, Z. Cheng, H.K. Tsang, G. Mashanovich, and G.T. Reed, "Suspended membrane waveguide on silicon-oninsulator for mid-infrared wavelengths," EOS Annual Meeting 2012, Aberdeen, UK, 25-28 September 2012.

[26] G. T. Reed, Silicon photonics: the state of the art (John Wiley \& Sons, Ltd, 2008).

[27] M. Nedeljkovic, T. B. Masaud, M. Strain, M. Sorel, M. M. Milošević, N. G. Emerson, G. T. Reed, H. M. H. Chong, and G. Z. Mashanovich, "Mid-infrared Mach-Zehnder and multimode interferometers in SOI," Opt. Lett. 2013.

[28] D. J. Thomson, Y. Hu, G. T. Reed, and J.-M. Fedeli, "Low loss MMI couplers for high performance MZI modulators," IEEE Photon. Technol. Lett. 22, 1485-1487 (2010).

[29] W. Bogaerts, S. K. Selvaraja, P. Dumon, J. Brouckaert, K. De Vos, D. Van Thourhout, and R. Baets, "Silicon-oninsulator spectral filters fabricated with CMOS technology," IEEE J. Sel. Top. Quantum Electron., vol. 16, pp. 3344,2010 